\title{
Erratum to: On the Optimal Transport of Semiclassical Measures
}

\section{Lorenzo Zanelli $^{1}$}

\section{Erratum to: Appl Math Optim DOI 10.1007/s00245-015-9317-1}

The author would like to correct the errors in the original publication.

In Eq. (1.10): Change sign of the potential from the wrong $+V(\gamma(t, \zeta))$ to the correct $-V(\gamma(t, \zeta))$.

In the inline equation, that is, four lines after Eq. (1.13): Change the wrong measure $\omega_{0}(x, p)=\delta\left(p-\nabla_{x} S_{+}(x)\right) \sigma_{0}(x)$ into the correct $\omega_{0}(x, p)=\delta(p-P-$ $\left.\nabla_{x} S_{+}(x)\right) \sigma_{0}(x)$.

The online version of the original article can be found under doi:10.1007/s00245-015-9317-1.

Lorenzo Zanelli

lorenzo.zanelli@unipd.it

1 Department of Mathematics, University of Padova, Via Trieste 63, 35121 Padua, Italy 